\title{
Detection of c-Ki-ras mutations in faecal samples from sporadic colorectal cancer patients
}

\author{
J Smith-Ravin, J England, I C Talbot, W Bodmer
}

\begin{abstract}
Colonic exfoliated epithelial cells in faecal material provide a source of human DNA which has been analysed for the presence of the tumour marker ras, in order to detect early tumour cells. The stool samples were subjected to a preliminary sample preparation step followed by centrifugation. DNA was extracted from both the centrifugation pellet and supernatant fractions, as well as from endoscopy washings, using a conventional phenol chloroform extraction method and was then purified on glass milk or spin columns. The purified DNA was amplified using mitochondrial primers and analysed for ras mutations using a nonradioactive, allele specific mismatch method. Corresponding tumour DNA was analysed for mutations using the same method. The results show that approximately $50 \%$ of the faecal samples analysed exhibited the presence of ras mutations which were also observed in the corresponding tumours. A double mutation was detected in one supernatant. Our findings represent an important stage in the development of a diagnostic test for the early detection of colorectal cancer.

(Gut 1995; 36: 81-86)
\end{abstract}

Keywords: colorectal cancer, c-Ki-ras mutation.

Colorectal cancer is one of the most common malignancies in the western world. Early diagnosis can lead to a cure by surgical procedures. At present, the only non-invasive test aimed at early diagnosis is the detection of faecal occult blood: the Haemoccult test. This test involves a chemical reaction to visualise blood released by a rectal or colonic tumour. However, it gives a high level of false positives because of the presence of dietary peroxidases and catalases or other interfering compounds in the stool samples. ${ }^{1}$ Bleeding may also result from non-cancerrelated conditions such as haemorroids; in addition, tumours less than $2 \mathrm{~cm}$ in diameter do not bleed enough to be detected. ${ }^{23}$ The sensitivity of Haemoccult has been reported to be only between 50 and $70 \%$ in patients with proved malignancies. ${ }^{34}$ Furthermore, there are insufficient data to indicate whether screening for colorectal cancer using the Haemoccult test reduces mortality from the disease in screened populations participating in clinical trials. ${ }^{4-6}$

The cells on the surface of the colonic mucosa are continuously being shed into the lumen and eliminated from the intestinal tract in the faeces. The renewal turnover in man is about $1 \%$ per hour and it has been calculated that the entire colon is renewed in three to four days. ${ }^{7}$ These colonic cells are rapidly destroyed in the faeces and, moreover, the presence of faecal material makes it impossible to detect abnormal cells by cytological methods. Rectal and colonic washings give a cleaner cell preparation and could be used for cytological analysis. ${ }^{910}$ In an earlier study, Raskin, ${ }^{11}$ using a cytological method, reported that over $90 \%$ of malignant tumours in the colon can be detected provided a sufficient number of well preserved malignant cells is present in the sample.

One of the sets of oncogenes most often associated with human neoplasia are the ras genes. The encoded oncoproteins (p21) are membrane-bound cytoplasmic proteins with both GTP-binding and GTPase activity, which are involved in cellular proliferation and differentiation. ${ }^{12}$ The oncogenic potential of the ras genes is activated by single base substitutions and the mutant forms of p21 participate in the development of a significant proportion of human cancers. ${ }^{13}$ In the case of colorectal cancer, the mutations span codons 12 and 13 of the c-KI-ras gene. These changes in the ras genes are an early step in carcinogenesis, and mutations are found to occur in at least 40 to $50 \%$ of colorectal adenomas and cancers. ${ }^{13-16}$ Previous reports show a correlation between tumour size and the presence of ras mutations. ${ }^{17}$

Recently, with the development of the polymerase chain reaction (PCR), a sensitive DNA amplification procedure, it has become possible to detect a few copies of target DNA in partially purified samples. ${ }^{18} 19$ The objective of our study is, as suggested by Bodmer, ${ }^{20}$ to investigate the application of PCR based techniques in the diagnosis of colorectal cancer with the aim of detecting the few abnormal cells which may be exfoliated from a small early malignant tumour. In addition, because ras mutations seem to occur during the early stages of carcinogenesis, ${ }^{141517}$ it would be of great medical interest if it were possible to detect the presence of ras mutations in faecal material such as stool specimens or endoscopy washings in patients at an early stage of the disease. This approach was first reported by Sidransky et $a l^{21}$ using an allele specific hybridisation method, while our studies were in progress.

A method was developed for extracting DNA from faecal material, followed by the use
Correspondence to: Cancer Research Fund, St Mark's Hospital, London Accepted for publication 23 March 1994 
of specific primers to amplify relevant DNA sequences from exfoliated colonic cells from sporadic colorectal cancer patients. Detection of human DNA was performed by PCR using primers specific for mitochondrial DNA sequences. Mitochondrial DNA molecules provide a convenient test for the quality control of DNA preparations from faecal samples since they are present at between a few and several hundreds of copies in eukaryotic cells, are highly variable between species and their sequences have been well established. ${ }^{22} 23$

We also investigated the presence of ras mutations in faecal samples obtained from sporadic colorectal cancer patients at different stages of the disease. Using an allele specific mismatch method, ${ }^{24}$ which has been shown to be sufficiently sensitive to detect one mutant cell out of $10^{4}$ cells in a mixture of normal and abnormal cells, ${ }^{25}$ we showed that it is possible to detect ras mutations in faecal samples from patients at different stages of the disease. This amplification method is based on efficient amplification of specific DNA templates that are in exact complementarity with their primers. Any single mismatch between the $3^{\prime}$ terminal base of the primers and the DNA templates will reduce or abolish amplification. Mutations in the corresponding tumours were confirmed by the same method. These results represent an important step in the development of a novel test for the detection of early stages of colorectal cancer.

\section{Methods}

\section{SPECIMENS}

The stool samples used in this study were collected from 11 sporadic colorectal cancer patients before undergoing colorectal surgery at St Mark's Hospital, London. Endoscopy washings were collected before biopsy from the same number of patients with colorectal disorders. Individuals who were found to be normal after endoscopy examination were used as controls. The faecal samples were collected in clean plastic containers which were given to each patient before bowel preparation.

\section{SAMPLE PREPARATION}

Although it was found that satisfactory DNA extracts could be prepared from stool samples stored at $-70^{\circ} \mathrm{C}$ for a few days, the samples were processed as soon as possible after collection according to the following procedure: 10 to $50 \mathrm{~g}$ of stool sample were diluted in $10-50$ $\mathrm{ml}$ of phosphate buffered saline (PBS), $\mathrm{pH} 7 \cdot 5$, and homogenised for 1 minute in a Stomacher 400 laboratory blender (Seward). The homogenate thus obtained was centrifuged for 10 minutes, at $2000 \mathrm{rpm}$, in a bench centrifuge and the resulting supernatant and pellet were used for the extraction of DNA according to the procedure below. Endoscopy washings $(100-200 \mathrm{ml})$ were immediately centrifuged and then subjected to the DNA extraction procedure. These washings contain colonic epithelial cells and residual faecal material and were obtained by flushing the bowel with water during endoscopy.

\section{DNA EXTRACTION}

The pellet fractions were resuspended in lysis buffer consisting of TE buffer (10 mM Tris, 1 mM EDTA, pH 7.5) containing $100 \mu \mathrm{g} / \mathrm{ml}$ proteinase $\mathrm{K}$ and $0.5 \%$ SDS. The supernatant fractions were supplemented with proteinase $\mathrm{K}$ and SDS to the same concentrations as above. Samples from both fractions were incubated at $55^{\circ} \mathrm{C}$ for several hours or overnight. In the case of malignant tissue, DNA was extracted from three, $10 \mu \mathrm{m}$ sections of frozen tumour tissue by overnight digestion with the lysis buffer as described above. Total DNA was purified from these samples by conventional phenol-chloroform extraction and ethanol precipitation. The ethanol-precipitated fraction was designated partially purified DNA. To remove contaminants and non-specific PCR inhibitors, the precipitate was resuspended in TE buffer, $\mathrm{pH}$ $7 \cdot 5$, or in sterile water and further purified, using glass milk (Bio 101, Stratech Scientific) or spin columns (Promega). Both products were used according to the manufacturers' instructions.

\section{AMPLIFICATION REACTION}

An aliquot $(5-10 \mu \mathrm{l})$ of the purified DNA was amplified by PCR in a DNA thermal cycler programmable heat block (Hybaid). Mitochondrial sequences, present at high copy number in eukaryotic cells, were detected using the pair of primers, L14841 and $\mathrm{H} 15149$, described by Scott et al. ${ }^{25}$ These primers are specific for the cytochrome $b$ gene. Another pair of primers, ND2 and COI, specific for the human mitochondrial cytochrome oxidase COI subunit, was also used (W Cui, personal communication).

The amplification reaction was carried out in a total volume of $50 \mu$ l of buffer containing $10 \mathrm{mM}$ Tris- $\mathrm{HCl}, \mathrm{pH} 8.8,1.5 \mathrm{mM} \mathrm{MgCl}_{2}, 50$ $\mathrm{mM} \mathrm{KCl}, 0 \cdot 1 \%$ Triton $\mathrm{X}-100,200 \mu \mathrm{M}$ dNTPs, $50 \mathrm{pM}$ of each primer and $1 \mathrm{U}$ of Taq DNA polymerase. The reaction mixture was covered with $50 \mu$ l of light mineral oil (Sigma Chemical Co, St Louis). The PCR conditions for the mitochondrial primers were as follows: denaturing temperature, $92^{\circ} \mathrm{C}$ for one minute; annealing temperature, $55^{\circ} \mathrm{C}$ for one minute and extension temperature, $72^{\circ} \mathrm{C}$ for three minutes. The amplification was carried out over 35 cycles.

The detection of ras mutations was performed using the allele specific mismatch method described by Stork et al. ${ }^{25}$ This method was used, after minor modifications, to detect mutations at the first and second base of codon 12 of the $\mathrm{Ki}$-ras gene; 40 to 50 amplification cycles were used. To validate the results obtained with the faecal samples, DNA from the corresponding tumours was used as control and amplification was per- 


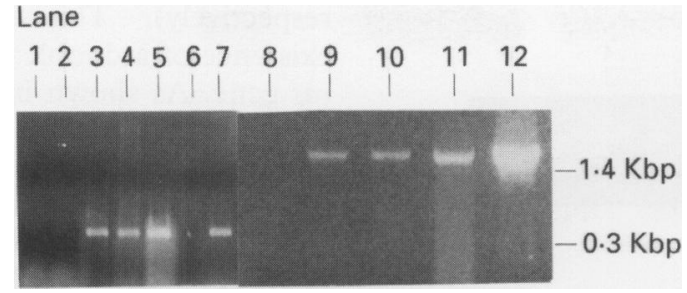

Figure 1: Amplification of DNA extracted from faecal samples (lanes $1,2,6,8)$ and endoscopy washings (lanes 3-5, 7, 9-12). A 300 bp PCR product was observed with H15149 and L14841 (lanes 1-7), and a 1.4 kbp with ND2 and COI (lanes 8-12).

formed under the same PCR conditions except that 35 cycles were used. The PCR products were analysed on a $1 \%$ or $3 \%$ agarose gel and visualised by ethidium bromide staining.

\section{PRIMERS}

The nucleotide sequences of the primers used in the amplification reactions are as follows:

L14841: 5' AAAAAGCTTCCATCCAA-

CATCTCAGCATGATGAAA $3^{\prime 26}$

H15149: 5' AAACTGCAGCCCCTCA-

GAATGATATTTGTCCTCA $3^{\prime 26}$

COI: 5' ACGATGTCTAGTGAT-

\section{GAGTTTGCTA $3^{\prime}$}

ND2: 5' ACGCCTAATCTACTCCACCTCAATC $3^{\prime}$

A primers:

K-ras A: 5' CAGAGAAACCTTTATCTG 3'

K-ras Aa: 5' TGGTAGTTGGAGCTA 3'

$\mathrm{K}$-ras Ac, K-ras Ag and K-ras At differ from $\mathrm{K}$-ras $\mathrm{Aa}$ in the last $3^{\prime}$ nucleotide which is replaced by $\mathrm{C}, \mathrm{G}, \mathrm{T}$ respectively. ${ }^{25}$

$B$ primers:

K-ras B: 5' GTACTGGTGGAGTATTT 3'

K-ras Ba: 5' ACTCTTGCCTACGCCAA 3'

$\mathrm{K}$-ras $\mathrm{Bc}, \mathrm{K}$-ras $\mathrm{Bg}$ and $\mathrm{K}$-ras $\mathrm{Bt}$ differ from

$\mathrm{K}$-ras $\mathrm{Ba}$ in the last $3^{\prime}$ nucleotide which is replaced by $\mathrm{C}, \mathrm{G} T$ respectively. ${ }^{25}$

\section{Results}

To detect the presence of specific human DNA sequences in faecal samples and endoscopy washings, human DNA was first isolated from the samples using a preparation method developed in our laboratory, followed by a conven-

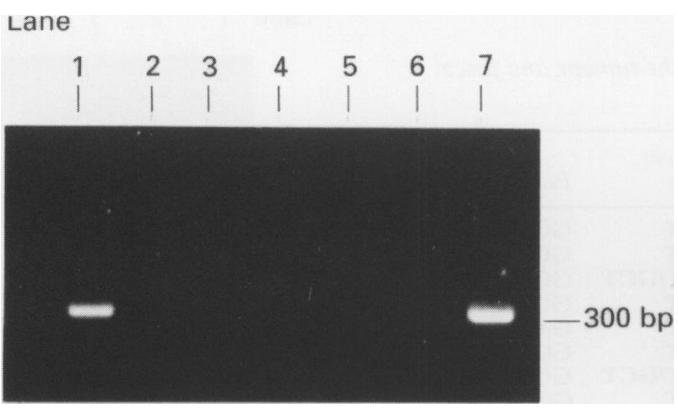

Figure 2: Amplification of phenol purified faecal DNA mixed with a known quantity of a purified amplifiable human colon DNA using the H15149 and L14841 primers. $A 300$ bp fragment is observed in lanes 1 and 7 . Lane 1: human colon DNA; lanes 2-6: mixture of human colon DNA and varying dilutions of faecal DNA (neat, 1:5, 1:100, 1:400, 1:800) of faecal DNA; lane 7: DNA $1: 5,1: 100,1: 400,1: 800)$ of faecal $D N A ;$
from endoscopy washing as positive control. tional DNA extraction procedure. These partially purified DNA samples were then used in amplification reactions with specific primers to detect mitochondrial sequences present at high copy number in eukaryotic cells. As shown in Figure 1, a 300 bp amplification product was detected in the endoscopy washings using H15149 and L14841 (lanes 3, 4, 5, 7) and a $1.4 \mathrm{kbp}$ fragment using ND2 and COI (lanes $9,10,11,12)$. In contrast, no band was observed for the partially purified faecal extracts (lanes 1, 2, 6, 8). To determine whether this lack of amplification was due to the presence of PCR inhibitors in the faecal samples, known amounts of purified amplifiable human DNA were added to the faecal extracts and the mixture was amplified under the same conditions. No amplification was observed for varying dilutions of faecal extract (Fig 2, lanes 2-6), thereby confirming the presence of PCR inhibitors in the faecal extracts. The extracts were therefore further purified using various procedures and then amplified using H15149 and L14841 (Fig 3). The best results were obtained on Bio 101 glass milk (lanes 2, 4, 6) and Promega spin columns (lanes $3,5,7$ ) both of which allowed the expected $300 \mathrm{bp}$ fragment to be observed. However, we found that spin columns gave consistently better purified DNA than glass milk. The former were therefore used for the purification of subsequent samples before analysis. No PCR product was observed in lanes 5 and 6 , due to excessive amounts of contaminant. Both of these samples gave the expected PCR band after subjecting them to a second purification under the same conditions.

To differentiate between normal and malignant cells, we investigated the presence of ras mutations in purified pellet and supernatant DNA fractions (Figs 4 and 5) using the allele specific mismatch method. This procedure involves the use of two sets of primers, $A$ and B, which are specific for mutations at the first and second base of codon 12 of the $\mathrm{c}-\mathrm{Ki}$ ras gene respectively. Each set contains one common primer and four other types of primers. The A set, which is specific for mutations at the first base of codon 12 , produces a $161 \mathrm{bp}$ amplification product. The $\mathrm{B}$ set generates a $146 \mathrm{bp}$ fragment which is used to determine the presence of mutations at the second base of codon 12. The $\mathrm{Ag}$ and $\mathrm{Bc}$

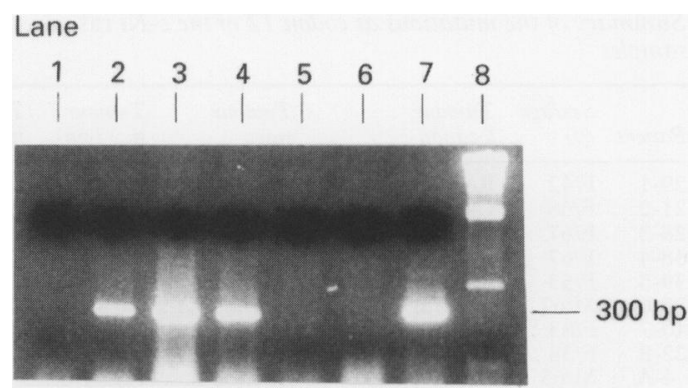

Figure 3: Amplification of faecal DNA purified using either glass milk (anes 2, 4, 6) or Promega spin columns (lanes 3, 5, 7). Lane 1: negative control. Lane 8: 1 Hind III molecular weight markers. Mitochondrial primers: H15145 and L14841. Samples in lanes 5 and 6 were found to be amplifiable after further purification under the same conditions. 


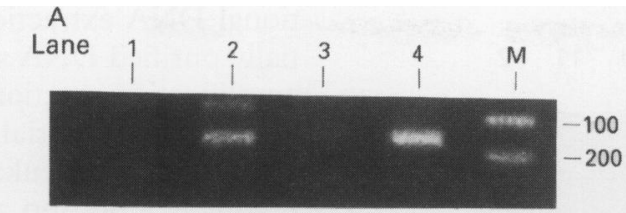

B

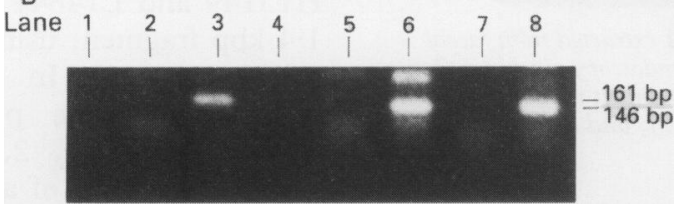

Figure 4: (1) Detection of ras mutations in DNA extracted from the pellet fraction of faecal samples from patient 39-5 using $B$ primers. $A 146$ bp band is observed with the $B$ wild type and Bt primers (lanes 2 and 4 respectively). Lanes 1 and 3 are $B a$ and $B g$ primers respectively. Lane M: 100 bp ladder marker. (2) Corresponding tumour $D N A$ was analysed with $A$ (lanes $1-4$ ) and $B$ primers (lanes 5-8). The mutation band present in the stool samples was also observed with the Bt primer (lane 8). No mutation band was observed with the $A$ primers. Lanes 3 and 6: $A g$ and $B c$ primers for wild type ras respectively.

primers are specific for the wild type sequence; the other primers are specific for various ras mutations.

Figure 4.1 shows the results obtained for stool samples from a colorectal cancer patient designated 39-5. Only the pellet fraction was initially analysed, using the $B$ primers. The presence of a $146 \mathrm{bp}$ fragment was observed with the Bc (wild type) and Bt primers (lanes 2 and 4 respectively).

To verify whether the mutation observed in the stool samples was also present in the corresponding colorectal tumour, we analysed tumour tissue from the same patient using both the A and B primers (Fig 4.2). No mutation band was observed with the A primers (lanes 1-4). On the other hand, the mutation band observed in the stool sample was also present in the tumour (lane 8). Lanes 5 and 7 show no band for $\mathrm{Ba}$ and $\mathrm{Bg}$ primers respectively.

Stool samples from another patient, 40-7, were also analysed using both the pellet and supernatant fractions (Fig 5.1, lanes 1-4 and 5-8 respectively); only the $B$ primers were used. In this case, one mutation band was observed with the pellet fraction (lane $3, \mathrm{Bg}$ primers) and two mutation bands with the supernatant fraction (lanes 5 and $7, \mathrm{Ba}$ and $\mathrm{Bg}$

Summary of the mutations at codon 12 of the $c-K i$ ras gene in the tumour and faecal samples

\begin{tabular}{clllllll}
\hline Patient & $\begin{array}{l}\text { Sex/age } \\
(y)\end{array}$ & $\begin{array}{l}\text { Tumour } \\
\text { location }\end{array}$ & $\begin{array}{l}\text { Tumour } \\
\text { stage }\end{array}$ & $\begin{array}{l}\text { Tumour } \\
\text { size }(\mathrm{cm})\end{array}$ & $\begin{array}{l}\text { Tumour } \\
\text { tissue }\end{array}$ & Pellet & Supernatant \\
\hline $29-1$ & F/42 & Rectum & Adenoc B & $3.5 \times 4 \cdot 0$ & GAT & GGT & GGT \\
$21-2$ & F/68 & Caecum & Adenoc C1 & $4 \cdot 0 \times 7 \cdot 0$ & GCT & GCT & GCT \\
$28-3$ & F/67 & Rectum & Adenoc B & $6 \cdot 5 \times 4 \cdot 0$ & GTT/GCT & GGT & GGT \\
$38-4$ & F/67 & Caecum & Adenoc C2 & $5 \cdot 0 \times 6 \cdot 0$ & GCT & GGT & GCT \\
$39-5$ & F/53 & Splenic flexure & Adenoc B & $6 \cdot 0 \times 8 \cdot 0$ & GAT & GAT & GAT \\
$35-6$ & M/67 & Rectum & Adenoc C1 & $6 \cdot 0 \times 8 \cdot 0$ & GAT & GGT & GGT \\
$40-7$ & F/83 & Rectum & Adenoc C1 & $7 \cdot 0 \times 6 \cdot 5$ & GTT/GCT & GCT & GTT/GCT \\
$22-8$ & F/38 & Ascending colon & Adenoc C1 & $4 \cdot 0 \times 5 \cdot 5$ & GGT & GGT & GGT \\
4-A & M/65 & Transverse & Adenoc B & $7 \cdot 5 \times 7 \cdot 0$ & - & GGT & GGT \\
5-A & F/63 & Tubular & Adenoc A & $4 \cdot 5 \times 3 \cdot 7$ & - & GAT & ND \\
34-A & M/52 & Tubular & Adenoc A & $2 \cdot 0 \times 3.0$ & - & GAT & ND
\end{tabular}

The mutations observed in the tumours, pellets and supernatants are presented under Tumour tissue, Pellet, and Supernatant respectively. These mutations were obtained as described in detail per cases 39-5 and 40-7. GGT is the expected wild type sequence. The mutated codons are in bold type. For example in patient 29-1, a mutation, GAT, was detected in the tumour bu not in either the pellet or supernant. $(-)=$ not available; $N D=$ not determined. respectively). This indicates the possible existence of a double mutation in the isolated ras gene. As shown in Figure 5.2, analysis of the corresponding tumour showed the presence of two mutation bands with the $B$ primers (lanes 5 and $7, \mathrm{Ba}$ and $\mathrm{Bg}$ respectively). No mutation band was observed with the A set of primers (lanes 1-4). This result is consistent with the result obtained from the stool sample supernatant.

A summary of our data is presented in the Table. Stool and tissue samples from a total of 11 patients were analysed for the presence of mutations. Codons in bold type indicate the mutations found in the tumours, and pellet and supernatant fractions. We were able to detect ras mutations in faecal samples from individuals with tumours localised as far as the caecum or as near as the rectum. Mutations were detected in stools from patients corresponding to tumours of all Dukes's stages A to C. Of the available tumour tissues analysed, only one did not show any mutation with both sets of primers.

\section{Discussion}

We have successfully amplified DNA extracted from faecal materials such as stool samples and endoscopy washings. Our data show that it is possible to detect human DNA sequences in faecal samples using PCR. Both sets of mitochondrial primers used allowed the correct PCR products to be observed. DNA extracted from endoscopy washings was suitable for amplification after phenol-chloroform purification and ethanol precipitation. However, PCR inhibition was observed when DNA extracted from stool samples was used as template in the amplification reactions. Wilde ${ }^{27}$ and $\mathrm{Xu}^{28}$ reported the presence of such PCR inhibitors during the isolation of viral RNA from faecal materials. No amplification was observed even

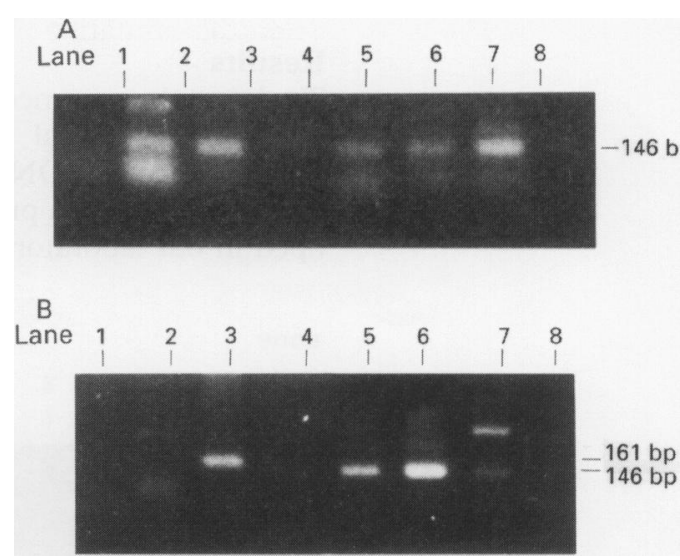

Figure 5: (1) Detection of ras mutations in DNA extracted from the pellet (lanes 1-4) and supernatant fractions lanes 5-8) of samples from patient 40-7. The amplification was performed on both fractions using the $B$ primers. The pellet shows one mutation band with the Bg primer (lane 3) whereas a double mutation band was observed in the supernatant with the $\mathrm{Ba}$ (lane 5) and $\mathrm{Bg}$ (lane 7) primers. Lane 2 and 6: $B c$ primers for wild type ras. (2) The presence of mutations in the corresponding tumour analysed using the $A$ (lanes 1-4) and $B$ (lanes 5-8) primers. $A$ double mutation was also observed with the $\mathrm{Ba}$ (lane 5) and Bg (lane 7) primers. No mutation band was observed with $A$ primers. Lanes 3 and 6: $A g$ and Bc primers for wild-type ras respectively. 
after diluting the samples up to 800 -fold. The inhibition may be due to a high concentration of contaminants which are not removed by the phenol extraction procedure. The use of Promega spin columns, designed for the purification of plasmid DNA, proved to be a rapid method for removing the PCR inhibitors without the need for elaborate sample preparation.

When the samples are centrifuged during the preliminary preparation procedure, intact cells are recovered in the pellet and DNA from lysed cells in present in the supernatant. Both types of DNA samples were amplified and used for the detection of ras mutations. We were able to amplify DNA extracted from the supernatant. This result is of great interest as it offers a simple method for the detection of human DNA sequences. Furthermore, analysis of the supernatant, in patient $40-7$, revealed a double mutation that was not detected in the pellet. The absence of the double mutation in the pellet DNA may be due to a high level of cell degradation, resulting in fewer cells in the pellet and a higher concentration of DNA in the supernatant.

Ras mutations were observed in about $50 \%$ of the faecal samples analysed. All the mutations detected in the faecal materials were found at the second base of codon 12, the majority $(86 \%)$ are mutations of $G$ to $A$ or $C$, which indicate changes from wild-type glycine to mutant alanine and aspartic acid. Sidransky et $a l^{21}$ have also reported the detection of ras mutations in faecal DNA isolated from pellets derived from stool samples using an allele specific hybridisation technique. In some cases, the mutations present in the tumours were not detected in the faecal samples (Table). This result may be due to a low copy number of ras genes in the samples. In addition, with such samples, the sensitivity of the method, and therefore the detection of very low yields of amplified product, may be decreased because of the complex nature of the samples.

All the mutations found in the tumours $(85 \%)$ were located at the second base of codon 12. These data are consistent with those previously reported. ${ }^{13}$ The tumour from patient 40-7 showed a double mutation at the second base of codon 12 and both mutations were found in the corresponding faecal sample. Farr et $a l^{29}$ observed a double mutation in individual polyps from a patient with familial adenomatous polyposis using a dot blot analysis. The double mutation observed might have arisen from two different malignant cell clones, but it is also possible that a second hit might have occurred in the original single clone at a later stage of development of the tumour. Our results show that tumours larger than 5.0 $\mathrm{cm}$ were detectable, irrespective of their location in the colon, and tumours in the caecum were detectable with the same probability as the ones in the rectum. We also observed mutations in two cases of early adenocarcinomas (Dukes's stage A). In the case of endoscopy washings, amplification of the wild type allele was observed with the A and B sets of primers in all the samples, but no ras mutations were detected. Polyps removed during the endoscopy examinations were found to be normal on pathological analysis.

Some non-specific, high molecular weight bands which were sometimes observed on agarose gel (Fig 4.2, lanes 5 and 6) may be due to the large number of amplification cycles and to the low annealing temperature used.

The detection of human DNA in faecal samples will greatly facilitate the detection of specific DNA sequences from exfoliated normal and abnormal cells of the intestinal tract. The method used to detect ras mutations is based on a non-radioactive allele specific amplification which has, in addition, been shown to be more sensitive ${ }^{30}$ than the allele specific hybridisation method. ${ }^{21}$ These results open the way for the use of exfoliated colonic cell DNA obtained by a non-invasive method in the detection of early stages of colorectal cancer. Mutations arise in at least $40 \%$ to $50 \%$ of colorectal cancer cases. ${ }^{1415} \mathrm{By}$ applying similar methodology to the simultaneous analysis of ras, p53, and APC mutations it should be possible to increase the proportion of colorectal cancers or, even better, precancerous adenomas amenable to detection by faecal testing. This will of course require further improvement of the technique presented here, especially to detect a wide range of mutations along the lengths of the APC and p53 genes. These results are very promising and more work is being carried out to increase the sensitivity of this non-radioactive detection method in order to allow the detection of adenomas and early stages of colorectal cancer.

The authors are grateful to Dr C Williams and his team for providing the endoscopy washings.

The findings presented here have been partially published in abstract form in Gut 1993; 34 (suppl 1): S53.

1 Macrae FA, St John JB, Caligiore P, Taylor LS, Legge JW. Optimal dietary conditions for hemoccult testing. Gastroenterology 1982; 82: 899-903.

2 Macrae FA, St John JB. Relationship between patterns of bleeding and hemoccult sensitivity in patients with colorectal cancers or adenomas. Gastroenterology 1982; 82: 891-8

3 Simon JB. Occult blood screening for colorectal carcinoma: a critical review. Gastroenterology 1985; 88: 820-37.

4 Macrae FA. Faecal occult blood testing: sensitivity and specificity. Br f Surg 1985; 72: S67-S74.

5 Castiglione G, Grazzini G, Poli A, Bonardi R, Ciatto S. Hemoccult sensitivity estimate in a screening program for Hemoccult sensitivity estimate in a screening program for colorectal cance

6 Mandel JS, Bond JH, Bradley M, Snover DC, Church TR, Williams $S$, et al. Sensitivity, specificity and positive predictivity of the Hemoccult test in screening for colorectal cancer. Gastroenterology 1989; 97: 597-600.

7 Lipkin M, Bell B, Sherlock P. Cell proliferation kinetics in the gastrointestinal tract of Man. I. Cell renewal in colon and rectum. $\mathcal{F}$ Clin Invest 1963; 42: 767-76.

8 Shorter RG, Moertel CG, Titus JL, Reitemeier RJ. Cell kinetics in the jujenum and rectum in Man. Am $\mathcal{F}$ Digestive Dis 1964; 9: 760-3.

9 Bader GM, Papanicolaou GN. The application of cytology in the diagnosis of cancer of the rectum, sigmoid and in the diagnosis of cancer of the rectum,
descending colon. Cancer 1952; 2: 307-14.

10 Prolla JC, Kirsner JB. Handbook and atlas of gastrointestinal exfoliative cytology. Chicago and London: University of Chicago Press, 1972

11 Raskin HF, Palmer WL, Kirsner JB. Exfoliative Cytology in diagnosis of cancer of the colon. Dis Colon Rectum 1959; 2: 46-57.

12 Polakis P, McCormick F. Interactions between p21 ras proteins and their GTPase activating proteins. Cancer Surveys 1992; 12: 25-41.

13 Capella G, Cronauer-Mitra S, Peinado MA, Perucho $M$. Frequency and spectrum of mutations at codons 12 and 13 of the c-K-ras gene in human tumors. Environm Health Perspect 1991; 93: 125-31.

14 Bos JL, Fearon ER, Hamilton Sr, Verlaan-de Vries M, van Boom JH, van der Eb A, Vogelstein B. Prevalence of ras 
gene mutations in human colorectal cancer. Nature 1987; 327: 293-7.

15 Forrester K, Almoguera C, Han K, Grizzle WE, Perucho M. Detection of high incidence of K-ras oncogenes during human colon tumorigenesis. Nature 1987; 327: 298-303.

16 Rodriguez NR, Rowan A, Smith MEF, Kerr IB, Bodmer WF, Gannon JV, et al. p53 mutations in colorectal cancer. Proc Natl Acad Sci USA 1990; 87: 7555-9.

17 Volgelstein B, Fearon ER, Hamilton SR, Kern SE, Preisinger AC, Leppert $\mathrm{M}$, et al. Genetics alterations during colorectal-tum

18 Gouvea V, Glass RI, Woods $\mathrm{P}$, Taniguchi $\mathrm{K}$, Clark HF, Forrester B, Fang Z. Polymerase chain reaction amplification and typing of Rotavirus nucleic acid from stool specimens. $\mathcal{F}$ Clin Microbiol 1990; 28: 276-82.

19 Gumerlock PH, Tang YJ, Meyers FJ, Silva J. Use of the polymerase reaction for the specific and direct detection of Clostridium difficile in human feces. Rev Infect Dis 1991 ; 13: $1053-60$.

20 Bodmer W. Hereditary colorectal cancer, a commentary. In: Utsunomiya J, Lynch T, eds. Hereditary colorectal cancer Berlin: Springer-Verlag, 1990.

21 Sidransky D, Tokino T, Hamilton SR, Kinzler KW, Levi B, Frost P, Vogelstein B. Identification of ras oncogen mutations in the stool of patients with curable colorecta tumors. Science 1992; 256: 102-5.

22 Anderson S, Bankier AT, Barrell BG, de Bruijn MHL Coulson AR, Drouin J, et al. Sequence and organization of the human mitochondrial genome. Nature 1981; 290: 457-64.
23 Brown WM. The mitochondrial genome of animals. In: Macyntire RJ, ed. Mol Evol Genetics. New York: Plenum Press, 1985: 95-130.

24 Newton CR, Graham A, Heptinstall LE, Powell SJ, Summers $\mathrm{C}$, Kalsheker $\mathrm{N}$, et al. Analysis of any point mutation in DNA. The amplification refractory mutation system (ARMS). Nucleic Acids Res 1989; 17: 2503-16.

25 Stork P, Loda M, Bosari S, Wiley B, Poppenhusen K, Wolfe $H$. Detection of K-ras mutations in pancreatic and hepatic Heoplasms by isotopic mismatched polymerase chain neoplasms by isotopic mismatche

26 Scott VE, Arctander P, Wilson AC. Mitochondrial resolution of the deep branch in the genealogical tre or perching birds. Proc $R$ Soc Lon Biol 1991; 243. 99-107.

27 Wilde J, Eiden J, Yolken R. Removal of inhibitory substances from human fecal specimens for detection of group A rotaviruses by reverse transcriptase and polymerase chain reaction. $\mathcal{F}$ Clin Microbiol 1990; 28: 1300-7. $28 \mathrm{Xu} \mathrm{L,} \mathrm{Harbour} \mathrm{D,} \mathrm{McCrae} \mathrm{MA.} \mathrm{The} \mathrm{application} \mathrm{of}$ polymerase chain reaction to the detection of rotaviruses polymerase chain reaction to the detection

29 Farr CJ, Marshall CJ, Easty DJ, Wright NA, Powell SC, Paraskeva C. A study of ras gene mutations in colonic adenomas from polyposis patients. Oncogene 1988; 3: 673-8

30 Smit VTHBM, Boot JMA, Smits AMM, Jan Flearen G, Cornelisse CJ, Bos JL. K-ras codon 12 mutations occur very frequently in pancreatic adenocarcinomas. Nucleic Acids Res 1988; 16: 7773-82. 